\title{
Energy aware cross layer-based connection admission control mechanism for wireless mesh networks
}

\section{Pradeep Reddy*}

School of Information Technology and Engineering, VIT University, Vellore,

Tamil Nadu, India

Email: pradeep@vit.ac.in

*Corresponding author

\section{P. Venkata Krishna}

Department of Computer Science,

Sri Padmavathi Mahila Visvavidyalayam Tirupati 517502,

AP, India

Email: dr.krishna@ieee.org

\begin{abstract}
This paper studies the allocation scheme of bandwidth for the purpose of a quality end-to-end communication in multi-hop and multi-radio wireless mesh networks. In wireless mesh networks the role of bandwidth in providing QoS is pivotal. In view of the limited availability, efficient allocation of bandwidth becomes necessary. We proposed a scheme for connection admission control based on energy aware cross layer mechanism, to address the problems relating to the allocation of bandwidth for real-time and non-real-time traffic utilising a shared database. The shared database contains the energy available at the node, routing information, prediction of congestion and issues of congestion control in order to maximise the throughput of the network. The performance of the proposed model is evaluated in comparison with the other models utilising cross layer techniques. The design of the proposed network developed on the basis of energy aware cross layer optimisation, which yields considerable enhancement in the throughput of the network and these results can be crucial in the practical design of the network.
\end{abstract}

Keywords: energy; cross layer optimisation; bandwidth allocation; congestion control.

Reference to this paper should be made as follows: Reddy, P. and Krishna, P.V. (2016) 'Energy aware cross layer-based connection admission control mechanism for wireless mesh networks', Int. J. Smart Grid and Green Communications, Vol. 1, No. 1, pp.73-86.

Biographical notes: Pradeep Reddy is currently working as an Associate Professor in SITE, VIT University, Vellore, India. He received his $\mathrm{PhD}$ degree and MTech in Computer Science and Engineering from VIT University India and BTech in Computer Science and Engineering from JNT University, Andhra Pradesh. His research interests include mobile and wireless systems and open source technologies. He is a member of various professional organisations such as IEEE, CSI and IAENG. He has published several papers in national and international journals and conferences. 
P. Venkata Krishna is working as a Professor, Computer Science Department, Sri Padmavathi Mahila Visvavidyalayam Tirupati, India. He received his BTech in Electronics and Communication Engineering from Sri Venkateswara University, Tirupathi, India, MTech in Computer Science and Engineering from REC, Calicut, India and PhD degree from VIT University, Vellore, India. His research interests include mobile and wireless systems and grid computing. He has published over 100 papers in national and international journals and conferences. He is a reviewer and editor for many reputed journals.

\section{Introduction}

The role of wireless mesh networks (WMNs) in the development of networks of the future generations is significant. The features of cost efficiency and faster configuration of these WMNs recommend themselves for their wider networking applications including campus or community networks. The major problem encountered by all these networks is bandwidth management. The architecture of (WMN) (Akyildiz et al., 2005, 2008; Krishna and Iyengar, 2007) consists of mesh clients and a wireless backbone with mesh routers and gateway nodes forming back-haul links in order to provide internet connectivity. The mesh clients in the context could be either mobile or stationary. WMNs are trusted to provide large coverage access to different services. The mesh routers could also be mobile, but our focus is on the infrastructure/backbone (Akyildiz et al., 2008) mesh network, which is a widely used network structure.

The variation in scale of access-networks consisting in internet today is very vast such as from tens of thousands of users supported through large wired access networks, wide area networks in campus or providers of internet services to comparatively much smaller wireless access-networks which can support only tens to hundreds of users. The interconnection of these access networks to the core networks can provide support to hundreds of millions of users. Primarily all these networks provide two categories of services namely, 'guaranteed service' and 'best effort service'. The networks in guaranteed service provide certain types of service guarantee to either individual users or groups of users. Often, such guarantees are to ensure that the throughput in the case of a group of users is higher than certain prescribed minimum value or that the delay which is experienced is always smaller than certain threshold value. Most of the times, the offered guarantees are in a statistical sense, e.g., giving a specific data rate $\mathrm{x} \%$ of the time. However, the network does not make any promises in the best effort service. A sort of 'elastic traffic' makes use of this typical service, where it is not obligatory for the users to have any specified minimum requirements, but instead try to get the maximum possible data to their respective destinations in the quickest time possible. The data flows of the individual users take cognisance of the congestion in the network and correspondingly react by adapting their rate of transmission, so that the congestion could be minimised. One such instance of providing the best effort service is email service in general. In WMN all the potential applications expect the network to efficiently deliver greater volume of traffic. Consequently, the primary design goal of the WMNs should be the ways and means to enhance the throughput of the network. Also, fairness must be considered in every context of throughput, or else, the result could be a serious and undesirable bias on the allocation of network resource. Some of the very fundamental 
issues to be considered in the management of bandwidth are, utilising bandwidth effectively through proper channel allocation, reducing the congestion levels, estimating and evaluating the available bandwidth at any point of time, monitoring of wireless channels, adapting the flow and rate and providing QoS guarantees (Akyildiz et al., 2008). We proposed a bandwidth aware cross layer scheme in this paper, which can address the issue of allocation of bandwidth for real time and non-real time traffic utilising a shared data base consisting of routing information, prediction of congestion and problems associated with congestion control, to maximise the network throughput. The proposed model collects and makes use of various metrics for different layers to form a shared database in such a way that any application, based on a type can effectively choose the available bandwidth.

The remaining paper is organised as detailed below:

Section 2 contains discussion of the related work and accomplishments in the relevant area till now. Section 3 illustrates the proposed system model. Section 4 projects a comparative analysis of our proposed approach and other existing approaches so far. Conclusions of the proposed work and the future possibilities are discussed in Section 5.

\section{Related works}

Several recent researches have been focusing on developing cross layer designs for various requirements of multi-hop wireless networks. Design of optimal routing solutions has been the prime concern of a majority of the proposed models. Only limited studies concentrated on developing the cross layer model, which is capable of estimating and controlling the congestion. In our model, we tried to give a shared database that contains application layer, transport layer, network layer and MAC layer. Cheng, et al. (2013) considered two diverse cross layer mechanisms utilised for the reduction of the delay in (WMC): a loosely coupled cross layer scheme (Cheng et al., 2013) that addresses the issues of routing and control of link rate and scheduling of link transmission, and a tightly coupled cross layer scheme (Cheng et al., 2013) that can optimise routing paths, link rates and assignment of time slots for individual transmissions. Separate optimisations are done for every individual sub-problem in the loosely coupled cross layer scheme. This is between the network layer and the MAC layer. MAC layer decisions regarding scheduling and route optimisation from network layer are treated as separate entities and addressed accordingly. Conversely, in the tightly coupled cross layer scheme the problems of both MAC layer and network layer are treated and optimised in one solution. Hou et al. (2012) proposed a routing mechanism with bandwidth guarantee for utilisation in wireless networks. The authors have introduced a novel new path weight parameter which can capture the available bandwidth in any specific route. Both route table and distance table are constructed on the basis of this available bandwidth and optimise the routing protocol. Cao et al. (2008) proposed a mechanism for (WMC) capable of maintaining the stream of data packets. An efficient CSMA/CA scheme is proposed by Zhao et al. (2013) for efficiency in both power and bandwidth. Ding et al. (2013) defined a solution optimised for on-demand video applications in the networks having high capacity The current model advocated a fusion of multi-path routing and allocation of spectrum to reduce the total cost of bandwidth in the network. In order to solve the problem of bandwidth, the authors proposed centralised and distributed channel 
allocation mechanisms. Huang et al. (2011) proposed an approach using cross layer to handle link asymmetry. This cross layer model is in between the MAC and network layers. For the purpose of handling the problems of both the hidden and exposed terminals an integrated mechanism for routing and channel allocation for unidirectional link has been proposed.

The proposed model utilises the network allocation vector (NAV) for monitoring and controlling the channel and the local routing spanning tree for routing and maintenance. Yazdanpanah et al. (2011) proposed a cross layer optimisation with smart antennas for WMN. The given model also studied a cross layer design in between MAC and physical layers and proposed a combined allocation of bandwidth and element scheduling. Pathak et al. (2011) has presented the problems of design in (WMC) in a survey on networks. In addition, the author also examined various joint design approaches and offered explanations of design issues independently. An optimised model is presented by Copane et al. (2010) for the purpose of routing, scheduling and assignment of channels. Here the author considered every technique of optimisation separately. He experimented with a joint routing and scheduling for single channel multiple radios. Tang et al. (2010) proposed a fair mechanism for the allocation of bandwidth using linear programming so that good trade off between fairness and throughput can be achieved. Fathi et al. (2010) proposed a cross layer joint rate control and scheduling for WMN. The three layers, transport layer, MAC layer and physical layer, are involved in the proposed cross layer technique. Here the transport layer handles the rate control, resource allocation is done by the MAC layer and the Physical layer makes use of OFDMA technique with beam foaming to provide scheduling. Cicconetti et al. (2009) proposed a mechanism to balance the bandwidth known as fair end-end bandwidth allocation (FEBA) which can be implemented at the MAC layer only for single radio multiple channels. The authors presented a round-robin mode for the purpose of granting and maintain requests and grant data structure. The quantity of service at each round corresponds to the incoming and outgoing flows. The one major setback of the model is its constraint to serve all categories of traffic, since its design factor addresses only single radio multiple channels. Shen et al. (2009) proposed an admission control mechanism on the basis of the estimation of available bandwidth. It estimates the available bandwidth on the basis of the ratio of channel traffic and the problem of hidden terminal. On estimating the bandwidth availability, a corresponding ACA is designed at the MAC layer. Rong et al. (2009) proposed an approach for connection admission control (CAC) in order to negotiate handoffs utilising mobile agents. CAC is implemented at mesh router with each client attached with a mobile agent (MA). The movement of the client results in corresponding migration of MA. MA can negotiate the hand-off supported with backup connection for handoff cells. Baldo (2009) proposed a cross layer distributed admission control mechanism (Rad and Wong, 2008; Wang et al., 2008; Ergin et al., 2008) for providing QoS in WMN. The authors proposed a joint routing and admission control mechanism (Ergin et al., 2008) by fusing various layers such as the network, MAC and physical layers. Rad and Wong (2008) proposed a cross layer fair bandwidth sharing algorithm for ensuring fairness in the allocation of channels by integrating channel assignment and interference range. Wang et al. (2008) proposed a joint bandwidth allocation, element assignment and link scheduling for utilisation in (WMC) having MIMO links. The proposal includes a cross layer optimisation (Yazdanpanah et al., 2011) process between the MAC and the physical layers in order to design a cost-aware-element assignment technique for the purpose of minimising the total number of antennas. Ergin et al. (2008) 
proposed two methods for the estimation of bandwidth availability, namely, dual-carrier sense with parallel transmission (Ergin et al., 2008) and packet probing with RTS/CTS Handshake (Ergin et al., 2008).

\section{System model}

In mobile Adhoc networks, the quality of service support depends on several factors such as on the network resources available and the rate of mobility of such resources and vastly varies from what can be obtained in fixed networks such as internet. Besides, potentially the (WMC) have limited resources in comparison to the fixed networks. Hence, multiple and complex criteria are needed to capture inter-node quality of the links, alternate and best paths, method of channel access and mechanisms of congestion and flow. Figure 1 displays the proposed model utilising back and forth information flow with two new interfaces created between the twin layers to enable information flow in both ways. Occurrence of this iterative loop between the two layers becomes possible only if the two layers perform diverse tasks, and are willing for a mutual collaboration during run time. The basis for the model is the break-up of the network features into four major modules namely; application layer metrics (ALM), transport layer metrics (TLM), network layer metrics (NLM) and MAC layer metrics (MLM). The ALM defines the category of the traffic type which the network uses. Congestion notification on the basis of ECN is defined by the TLM. Utilising random early detection (RED), this metric provides information of buffers and about congestion. Best path and alternate path information is provided by the NLM. Lastly, the MLM are concerned about the estimation of the bandwidth and delay information. The next section illustrates the CLCAC which makes interactions among various layers.

The four structures of message above are useful for the analysis of the network condition in such a way that there is a significant increase in the performance of the wireless network by a process of sharing metric information optimally, through a common database. The common database will consider different factors such as energy at the node, Available bandwidth in the path and also number of hops in the path.

\subsection{MAC layer metric structure}

Storing of available bandwidth information at each node and estimation of available bandwidth for the specific paths are done by MLM structure.

The MLM message structure can be defined as follows.

\subsubsection{MLM (path, delay, bandwidth estimation)}

Calculations of the routes from single source to single destination and from multiple sources to multiple destinations are taken up first. On obtaining the routes we can perform the estimation of bandwidth (Reddy and Krishna, 2012; Krishna et al., 2008; Reddy et al., 2014; Krishna et al., 2009; Misra et al., 2010; Krishna et al., 2012; Misra et al., 2013; Krishna et al., 2008; Misra et al., 2014) for the specific routes in order to discover the optimal path with more bandwidth availability. The MLM layer then compares the required bandwidth of the application to the available bandwidth of different routes. Based on these comparisons it chooses the optimal path based on 
application requirements. Such selection will utilise the bandwidth effectively as it is allotted based on user requirement. Once MLM gets the optimal path then the packets are routed in this optimal path.

Figure 1 CLCAC through a shared database (see online version for colours)

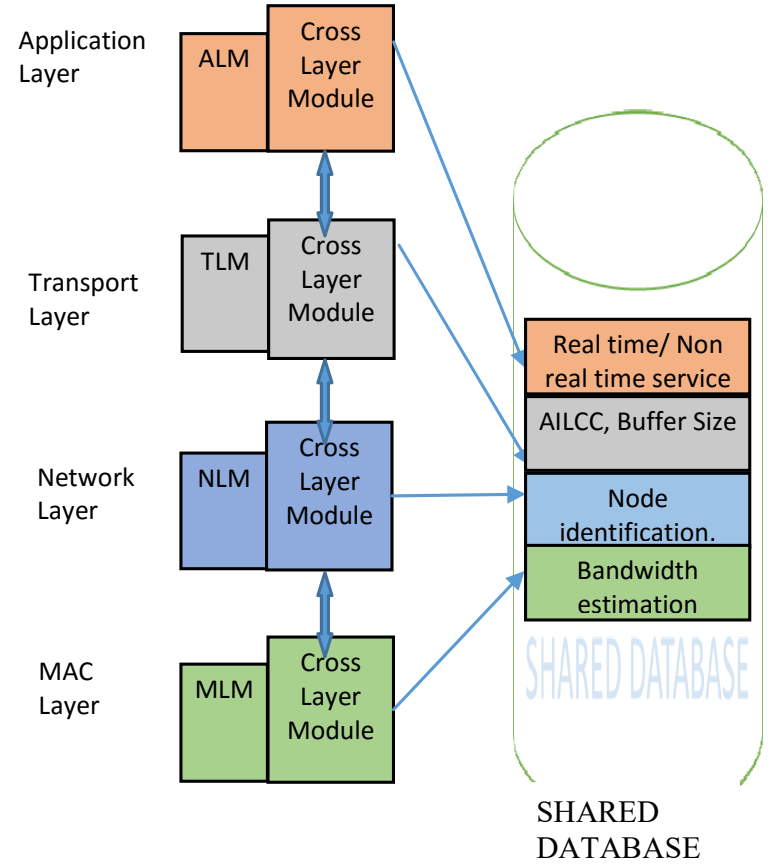

\subsection{Network layer metric structure}

NLM structure stores the relevant information about various issues in the network such as node information and metrics of path selection such as delay and bandwidth. The NLM message structure can be defined as follows.

\subsection{NLM (node identification, route table)}

The domain of the route table is (mobility, BR)

The domain of the node identification could be a combination of characters and integers.

The node identification process is adopted with certain alterations from i.e., hash table node identification (HTNI) (Reddy and Krishna, 2012) method. In HTNI method the source mesh router computes the router ID of backbone router (BR) containing the ID of destination mobile node. The (BR) keeps track of the locations of its mobile nodes. BR sends a NSREQ (Node Search Request) if the mobile node is out of region which contains ID of BR and destination mobile id and sequence of packet. On finding the node the corresponding mesh router responds by sending its own router ID in the NSREP (Node Search Reply) packet to the BR. The packets are then routed to that BR (Reddy and Krishna, 2012; Krishna et al., 2008; Reddy et al., 2014; Krishna et al., 2009; Misra 
et al., 2010; Krishna et al., 2012; Misra et al., 2013; Krishna et al., 2008; Misra et al., 2014) by utilising the optimal path (Reddy and Krishna, 2012) through shared database (Krishna et al., 2008). The packets are further forwarded towards the destination mobile nodes after they reach the respective BRs. IN case of node failure the HTNI method is called automatically to find out the new optimal path.

\subsection{Transport layer metric structure}

TLM structure stores the necessary information regarding the issues of transport such as the status of congestion in the network. The TLM message structure can be defined as follows.

\subsubsection{TLM (AILCC, buffer size)}

AILCC involves six header bits:

- $\quad$ sender marks 'ECN-capable' when sending, ECT $=1$

- if router sees 'ECN-capable' and experiencing congestion, router marks packet as 'ECN congestion experienced', $\mathrm{CE}=1$

- if receiver sees 'congestion experienced', marks 'ECN echo' and CRP bits flag in responses until congestion $\mathrm{ACK}$ ' $\mathrm{d}, \mathrm{ECE}=1$ and $\mathrm{CRP}$ bits based on pheromone value

- if sender sees 'ECN echo', it reduces cwnd and marks 'congestion window reduced' flag in next TCP packet, CWR $=1$.

The domain of AILCC is $\{C R P, E C E$, and $C W R\}$ and the domain of the buffer size is integer.

Several network issues have been effectively addressed and solved through a nature inspired technique called ant colony optimisation. Ants release pheromone when they traverse through a specific path. The proposed model considered this pheromone value to estimate congestion and to offer an efficient congestion control mechanism. The presence of more pheromone value at nodes in any given network is a clear indication of more packets traversing in that path with a possibility of causing of congestion. The cause of congestion is indicated through a threshold pheromone value Pth (Reddy et al., 2014). It can be construed that the link overflow is the cause of congestion if pheromone value $\mathrm{P}$ happens to be more than Pth. Congestion at the link can be ruled out if the pheromone value happens to be less than the Pth and the cause of congestion here could be node failure.

If congestion occurrence can be predicted and determined, then we can initiate effective congestion control mechanisms.

The parameters of values of congestion rate with pheromone value (CRP) (Reddy et al., 2014), congestion window rate (CWR) and explicit congestion notification echo (ECE) can be the basis for identifying the level of congestion. The congestion window size is doubled when the packets are sent without the occurrence of congestion. There is always a direct correspondence between congestion levels in the network and the changes in the congestion window size.

The data is properly catalogued and due acknowledgements are dispatched to the sender when the packet is received successfully. The sender promptly deletes all 
corresponding data from the catalogue after receiving the acknowledgement for such sent packets. In the event of any packets being lost for any reason the sender retransmits the lost packets duly setting with retransmission bit set. The retransmission process without proper acknowledgements is termed as 'timeout retransmission'. The size of the congestion window doubles with every successful retransmission, which is an indication that the sender utilises more bandwidth in retransmitting the packets to the receiver.

The sender verifies for CRP bit after receiving the acknowledgement having ECN-Echo flag set. The congestion window size reduces corresponding to the values of CRP (Reddy et al., 2014). In the event of the CRP bits being 00, 01, 10 and 11 the reduction rate of congestion window size is $12 \%, 25 \%, 37 \%$ and $50 \%$ respectively. Then, the subsequent packets are set with CWR bit with modified threshold values which indicate the receiver about the proper response to the notification about congestion.

The receiver discontinues transmitting the packets having ECE bit set when such packets having CWR bit set are received.

\subsection{Application layer metric structure}

The ALM structure stored in shared database offers information about QoS by accessing other metric information. The ALM message structure is defined as follows.

\subsubsection{ALM (service category)}

The domain of the service category in ALM structure is \{real time/non-real time\}.

The call admission control initiates the required measures for the admission of the call based on the type of traffic such as real-time or non-real-time traffic. The optimal path having the mechanism of congestion control is selected for admitting the real-time traffic with full allocation of bandwidth because the real-time traffic is considered and treated as flow of high priority. Similarly, paths with moderate levels of congestion are used for admission of non real-time traffic and in incidents of high congestion levels.

Finally, when a path is selected for data transmission, the congestion window size of each node along the selected path is maintained as unique. Packet drops can be avoided by maintaining smaller size queue. If the uniqueness of the congestion window size of all the nodes is not maintained, then congestion could occur because of the possible variation of the bandwidth of the distinct edges from one another. When the bandwidth of the before edge is more, the sending rate of that node is considered to be high and more number of packets will arrive at such nodes with low sending rate and at this juncture the possibility of congestion occurrence enhances. Hence, maintenance of a unique congestion window size becomes imperative to assure that the sending rate of all the nodes is similar.

Figure 2 displays the bandwidth allocation model, illustrating the utilisation of available bandwidth for different types of calls.

It is necessary to specify an upper bound to the real-time applications in their utilisation of bandwidth to provide sufficient bandwidth required for non-real-time traffic. $B_{r \text { max }}$ represents the maximum bandwidth that is utilised by real-time traffic. Effective bandwidth allocations are provided in the following calculations.

$$
\begin{aligned}
& B_{t s h}=B_{A i} * 0.9 \\
& B_{r t \max }=B_{t s h} * 0.8
\end{aligned}
$$




$$
\begin{aligned}
& B_{n r t \min }=B_{t s h} * 0.2 \\
& B_{n r t \max }=B_{t s h} * 0.4 \\
& C m s g=B_{A i}-B_{t s h}
\end{aligned}
$$

Figure 2 Bandwidth allocation model (see online version for colours)

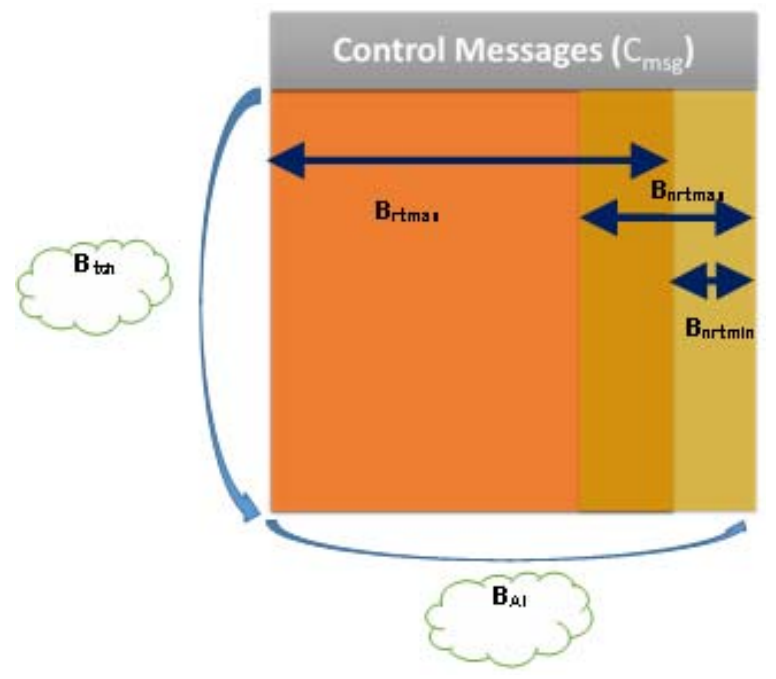

In the proposed model, we set $B_{r t \max }$ to be $80 \%$ of the bandwidth threshold value, which is denoted by $B_{t s h}$, and $B_{t s h}$ is $90 \%$ of the available bandwidth $B_{A i}$. This way, we can guarantee that the non-real-time traffic can occupy at least $20 \%$ of $B_{t s h}$ and at max of $40 \%$ of $B_{t s h}$. Max value subjected to the usage of bandwidth by real-time applications. $\left(B_{A i}-\right.$ $\left.B_{t s h}\right)$ is reserved for control packets and the route discovery packet flows. Table 1 projects the utilised simulation parameters.

\section{Performance evaluation}

Performance of the proposed model is provided with the utilisation of NS-2. NS-2 (network simulator 2) is a widely utilised open source wireless network simulator popular among academia.

The following metrics are utilised to estimate the performance of the proposed mechanism:

- Packet delivery throughput: this can be broadly explained as the quantity of data packets received at the destinations in correspondence to the packets dispatched at the sources.

- End-to-end delay of data packets: this illustrates the possible occurrence of delay between the originating time of the packet at the source and its arrival time at the source. Data packets lost during transit are not considered here. However, the delay 
metric properly considers the delays occurring due to route discovery, queuing and retransmission.

Table 1 Simulation parameters

\begin{tabular}{|c|c|c|}
\hline Duration & 100 & Length of measured simulation (seconds) \\
\hline Rate & 1,600 & Number of new connections/second \\
\hline Packmime-count & 1 & Number of pack mime clouds \\
\hline Queue-method & RED & Drop tail, RED, ... \\
\hline Queue-length & 10 & Queue length (packets) \\
\hline trg-delay & 0.001 & $\begin{array}{l}\text { Targeted delay at the RED queue } \\
\quad(\text { make min_thresh }=8 \mathrm{pkt})\end{array}$ \\
\hline Bytes & 0 & Packet-AQM \\
\hline Link-delay & $2 \mathrm{~ms}$ & Propagation delay on bottleneck link \\
\hline Link-bw & $2 \mathrm{Mb}$ & Bandwidth on bottleneck link \\
\hline Access-bw & $10 \mathrm{Mb}$ & Access link bandwidth \\
\hline Access-delay & $5 \mathrm{~ms}$ & Propagation delay of the access link \\
\hline Routers & 1 & Use routers/delay boxes in network \\
\hline Routing protocol & AODV & Use AODV as routing protocol \\
\hline Ecn & 1 & ECN enable by default \\
\hline ecn-syn & 0 & No ECN in SYN/ACK pkt \\
\hline ecn-syn-wait & 0 & ECN+ with no waiting \\
\hline Min rto & 0.2 & Min rto for TCP \\
\hline rtxcur-init & 3.0 & Initial value of RTO \\
\hline Init_window & 150 & Initial TCP window size (packets) \\
\hline Window & 500 & Max TCP window size (packets) \\
\hline Segsize & 750 & Default TCP segment size (bytes) \\
\hline Loss-rate & 0 & Maximum packet loss rate \\
\hline Run & 1 & Experiment run number \\
\hline Debug & 0 & Debug level \\
\hline Quiet & 0 & Don't output status messages \\
\hline Datadir & data & Directory to store output \\
\hline Make-trace & 1 & Make trace file \\
\hline http-trace & 1 & Trace HTTP connections \\
\hline rsp-size-avg & 3,000 & Average response size in bytes \\
\hline
\end{tabular}

\section{Results and discussion}

The results obtained amply prove the efficacy of CLACC cross layer-based connection admission control scheme in efficiently allocating bandwidth for both real-time and non-real-time applications duly reducing the congestion and increasing the packet flow in 
the network. Transmission of the packets from the source to destination and the loss of packets are computed by using the trace graph of the simulator. Performance of the sent packets, received packets and the lost packets is calculated in correspondence to the data transmission time and speed depending on the constant bit rate and its capacity of packet transmission.

Figure 3 Comparison of delay with and without CLACC (see online version for colours)

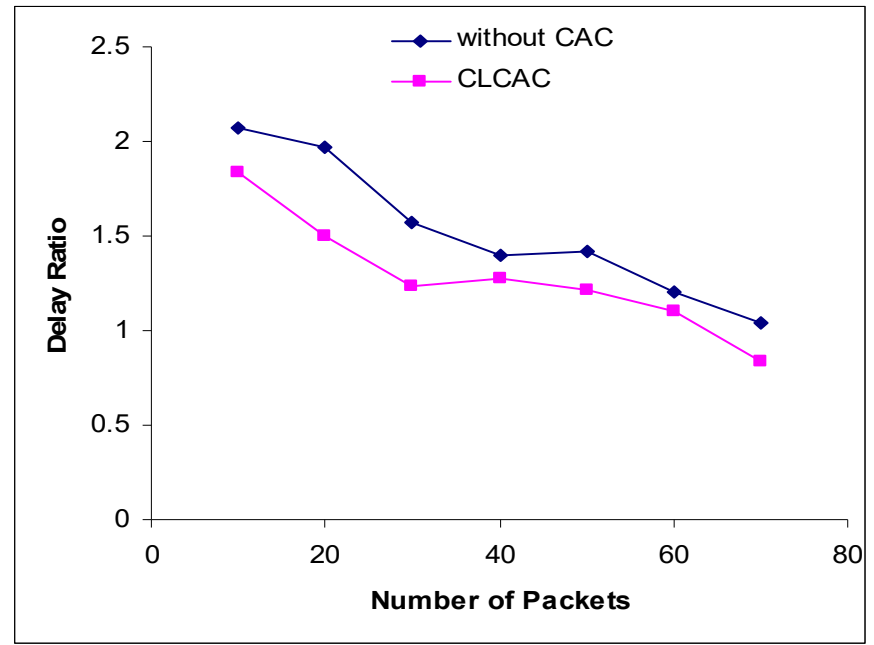

Figure 3 illustrates the delay performance of the proposed CLACC. The delay ratio can be obtained by estimating the source-to-destination packet delivery. Figure 3 illustrates that the delay ration with CLCAC is lesser, while it is noticed to be very high with out connection admission control.

Figure 4 Throughput in network with CLCAC (see online version for colours)

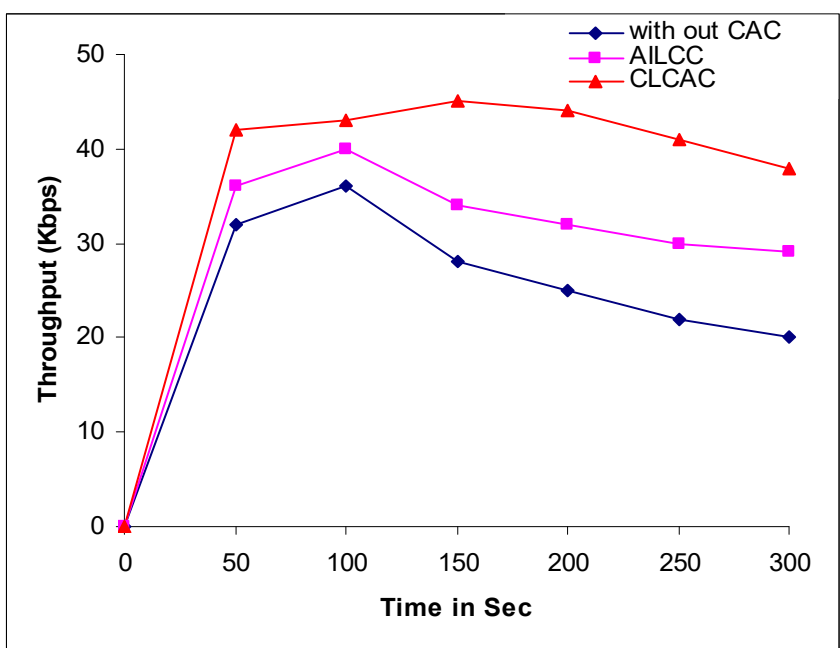


Figure 4 displays the graph of throughput performance in case of the proposed system by a comparison with both AILCC mechanism and without the mechanism of Connection Admission control. Any sudden drop in throughput is well prevented by CLCAC through effective utilisation of the available bandwidth for applications of both real-time and non-real-time.

Figure 5 illustrates the throughput for both real-time and non-real-time network traffic. When the throughput reaches certain threshold value, stability is noticed in it on comparison either with or without CLCAC. Once this has been established, a stable throughput can be guaranteed for each real-time flow.

Figure 5 End to end throughput (see online version for colours)

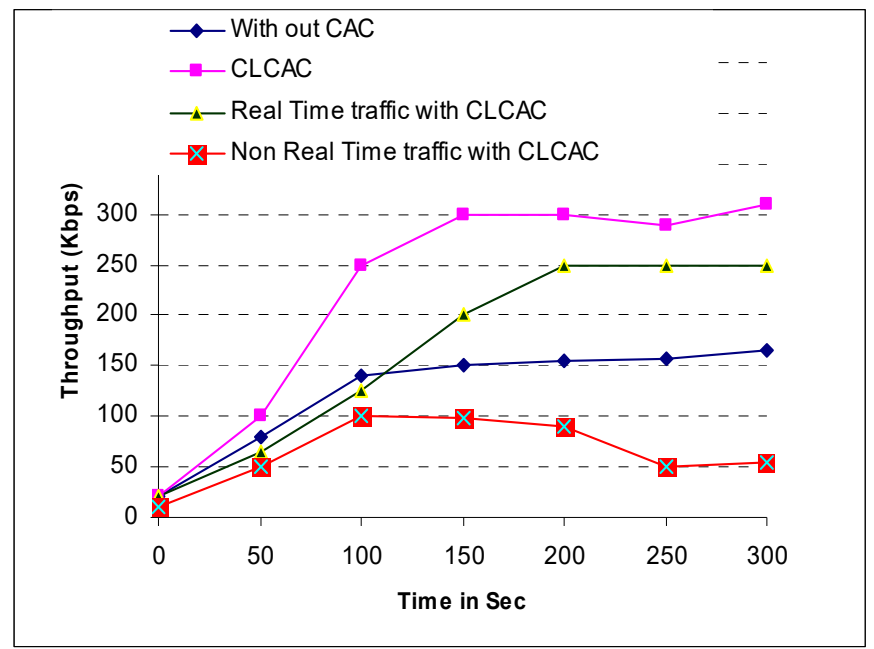

\section{Conclusions and future work}

We have proposed an effective mechanism for efficient allocation of bandwidth for different applications in WMNs. We have discussed in this paper, the mechanism of energy aware connection control admission based on cross layer which can be applied for effective utilisation of bandwidth. Appending to this improvisation, our proposed model also attempts to provide a quality aware shared database among the cross layer design with the following metrics such as the estimation of available energy at the node, bandwidth, identification of the node, routing table, mechanism for the control of congestion and mechanism for control of connection admission. Our proposed model also offers effective bandwidth utilisation for applications of real-time as well as non-real-time. In comparison with the existing models, our proposed model on analysis and evaluation is proved to provide more effective results. We intend extending this study in future in order to solve the starvation of applications by providing an effective queuing methodology. 


\section{References}

Akyildiz, I.F., Fellow, IEEE, Wang, X. and Member IEEE (2008) 'Cross-layer design in wireless mesh networks', IEEE Trans. Vehicular Technology, March, Vol. 57, No. 2, pp.1061-1076.

Akyildiz, I.F., Wang, X. and Wang, W. (2005) 'Wireles mesh network: a survey', Computer Networks, March, Vol. 47, No. 4, pp.445-487.

Baldo, O. (2009) 'A cross-layer distributed call admission control', Wireless and Mobile Computing, Networking and Communications, 2009. WIMOB 2009. IEEE International Conference on, 12-14 October, pp.441-446.

Cao, Y., Leung, K-C. and Li, V.O.-K. (2008) 'Bandwidth-guaranteed fair scheduling with effective excess bandwidth allocation for wireless networks', Wireless Communications, IEEE Transactions on, June, Vol. 7, No. 6, pp.2094-2105, doi: 10.1109/TWC.2008.05650.

Capone, G., Carello, I., Filippini, S. and Gualandi, F., Malucelli (2010) 'Routing, scheduling and channel assignment in wireless mesh networks: optimization models and algorithms', Ad Hoc Networks, August, Vol. 8, No. 6, pp.545-563.

Cheng, M., Ye, Q. and Cai, L. (2013) 'Cross-layer schemes for reducing delay in multihop wireless networks', Wireless Communications, IEEE Transactions on, February, Vol. 12, No. 2, pp.928-937, doi: 10.1109/TWC.2012.121412.120635.

Cicconetti, C., Akyildiz, I.F. and Lenzini, L. (2009) 'FEBA: a bandwidth allocation algorithm for service differentiation in IEEE 802.16 wireless mesh networks', IEEE/ACM Trans. on Networking, June, Vol. 17, No. 3, pp.884-897.

Ding, Y. and Xiao, L. (2013) 'Video on-demand streaming in cognitive wireless mesh networks', Mobile Computing, IEEE Transactions on, March, Vol. 12, No. 3, pp.412-423, doi: 10.1109/TMC.2011.273.

Ergin, M.A., Gruteser, M., Luo, L., Raychaudhuri, D. and Liu, H. (2008) 'Available bandwidth estimation and admission control for QoS routing in wireless mesh networks', Computer Communications, 9 May, Vol. 31, No. 7, pp.301-1317.

Fathi, M., Taheri, H. and Mehrjoo, M. (2010) 'Cross-layer joint rate control and scheduling for OFDMA wireless mesh networks', Vehicular Technology, IEEE Transactions on, October, Vol. 59, No. 8, pp.3933-3941.

Hou, R., Lui, K-S., Baker, F. and Li, J. (2012) 'Hop-by-hop routing in wireless mesh networks with bandwidth guarantees', IEEE Transactions on Mobile Computing, February, Vol. 11, No. 2, pp.264-277, doi:10.1109/TMC.2011.25.

Huang, Y., Yang, X., Yang, S., Yu, W. and Fu, X. (2011) 'A cross-layer approach handling link asymmetry for wireless mesh access networks', Vehicular Technology, IEEE Transactions on, March, Vol. 60, No. 3, pp.1045-1058.

Krishna, P.V. and Iyengar, S.N. (2007) 'A cross layer based QoS model for wireless and mobile Ad hoc networks', Journal of Mobile Communication, Vol. 1, No. 4, pp.114-120.

Krishna, P.V., Iyengar, N.C.S. and Misra, S. (2008) 'An efficient hash table-based node identification method for bandwidth reservation in hybrid cellular and Ad-hoc networks', Computer Communications, Vol. 31, No. 4, pp.722-733.

Krishna, P.V., Iyengar, N.Ch.S.N. and Misra, S. (2008) 'An efficient hash table-based node identification method for bandwidth reservation in hybrid cellular and Adhoc networks', Computer Communications, Vol. 31, No. 4, pp.722-733.

Krishna, P.V., Misra, S., Obaidat, M.S. and Saritha, V. (2009) 'An efficient approach for distributed dynamic channel allocation with queues for real-time and non-real-time traffic in cellular networks', Journal of Systems and Software, Vol. 82, No. 7, pp.1112-1124.

Krishna, P.V., Saritha, V., Vedha, G., Bhiwal, A. and Chawla, A.S. (2012) 'Quality-of-serviceenabled ant colony-based multipath routing for mobile ad hoc networks', IET Communications, Vol. 6, No. 1, pp.76-83. 
Misra, S., Krishna, P. V., Saritha, V., Agarwal, H. and Ahuja, A. (2014) 'Learning automata-based multi-constrained fault-tolerance approach for effective energy management in smart grid communication network', Journal of Network and Computer Applications, September, Vol. 44, pp.212-219.

Misra, S., Krishna, P.V., Abraham, K.I., Sasikumar, N. and Fredun, S. (2010) 'An adaptive learning routing protocol for the prevention of distributed denial of service attacks in wireless mesh networks', Computers \& Mathematics with Applications, Vol. 60, No. 2, pp.294-306.

Misra, S., Krishna, P.V., Saritha, V. and Obaidat, M.S. (2013) 'Learning automata as a utility for power management in smart grids', Communications Magazine, IEEE, Vol. 51, No. 1, pp.98-104.

Pathak, P.H. and Dutta, R. (2011) 'A survey of network design problems and joint design approaches in wireless mesh networks', Communications Surveys \& Tutorials, IEEE, Vol. 13, No. 3, pp.396-428, Third Quarter.

Rad, A.H.M. and Wong, V.W.S. (2008) 'Cross-layer fair bandwidth sharing for multi-channel wireless mesh networks', Wireless Communications, IEEE Transactions on, September, Vol. 7, No. 9, pp.3436-3445.

Reddy, C.P. and Krishna, P.V. (2012) 'An efficient bandwidth management framework for wireless mesh networks', Innovative Computing Technology (INTECH), 2012 Second International Conference on, 18-20 September, pp.102-106.

Reddy, Ch., Venkata, P. and Krishna, P. (2014) 'Ant-inspired level-based congestion control for wireless meshes networks', International Journal of Communication Systems, January 9th, Vol. 28, No. 8, pp.1493-1507, DOI: 10.1002/dac.2729.

Rong, B., Qian, Y., Lu, K., Hu, R.Q. and Kadoch, M. (2009) 'Mobile-agent-based handoff in wireless mesh networks: architecture and call admission control', Vehicular Technology, IEEE Transactions on, October, Vol. 58, No. 8, pp.4565-4575.

Shen, Q., Fang, X., Li, P. and Yuguang Fang, Y. (2009) 'Admission control based on available bandwidth estimation for wireless mesh networks', Vehicular Technology, IEEE Transactions on, June, Vol. 58, No. 5, pp.2519-2528.

Tang, J., Hincapie, R., Xue, G., Zhang, W. and Bustamante, R. (2010) 'Fair bandwidth allocation in wireless mesh networks with cognitive radios', Vehicular Technology, IEEE Transactions on, March, Vol. 59, No. 3, pp.1487-1496.

Wang, J., Du, P., Jia, W., Huang, L. and Li, H. (2008) 'Joint bandwidth allocation, element assignment and scheduling for wireless mesh networks with MIMO links', Computer Communications, 9 May, Vol. 31, No. 7, pp.1372-1384.

Yazdanpanah, M., Assi, C. and Shayan, Y. (2011) 'Cross-layer optimization for wireless mesh networks with smart antennas', Computer Communications, 1 October, Vol. 34, No. 16, pp.894-1911.

Zhao, L., Gao, L., Zhao, X. and Ou, S. (2013) 'Power and bandwidth efficiency of wireless mesh networks', Networks, IET, September, Vol. 2, No. 3, pp.131-140, doi: 10.1049/ietnet.2012.0061. 